\title{
Model Reduction Techniques for Frequency Averaging in Radiative Heat Transfer
}

\author{
René Pinnau and Alexander Schulze \\ Fachbereich Mathematik \\ Technische Universität Kaiserslautern \\ D-67653 Kaiserslautern, \\ Germany \\ email: \{pinnau, schulze\}@mathematik.uni-kl.de
}

\begin{abstract}
We study model reduction techniques for frequency averaging in radiative heat transfer. Especially, we employ proper orthogonal decomposition in combination with the method of snapshots to devise an automated a posteriori algorithm, which helps to reduce significantly the dimensionality for further simulations. The reliability of the surrogate models is tested and we compare the results with two other reduced models, which are given by the approximation using the weighted sum of gray gases and by an frequency averaged version of the so-called $\mathrm{SP}_{n}$ model. We present several numerical results underlining the feasibility of our approach.
\end{abstract}

Key words. Radiative Heat Transfer, Frequency Averaging, Proper Orthogonal Decomposition, Weighted Sum of Grey Gases, $\mathrm{SP}_{n}$ Approximations.

AMS(MOS) subject classification. 35K55, 49K20, 80A20

\section{Introduction}

The simulation of industrial high temperature processes requires to take into account heat conduction and convection as well as heat transfer via radiation, e.g. in simulation of gas turbine combustion chambers [24, 23], combustion in car engines or cooling of a hot glass melt $[4,26]$. Since the radiation field is dependent on time and space as well as on frequency and the angular direction, a simulation using a full radiative heat transfer model is computationally expensive; if the simulation is part of an optimization problem, it becomes infeasible $[2,14,5,19,21,20]$. In order to decrease the dimensionality, several simplified models have been developed, among them the Rosseland, $\mathrm{P}_{n}$ and $\mathrm{SP}_{n}$ equations that replace directed radiation by a direction-independent radiative flux $[17,11]$. The discretization with respect to frequencies is done by frequency band models; the so-called grey model is a model with just one band. Another possibility to reduce the high dimensional discrete phase space is to use adaptive discretization techniques [9].

Realistic simulation of the cooling of glass or combustion has to take into account that some frequencydependent properties of the material show rapid variations even on small frequency intervals; these 
rapid variations are also observed in experimental data or high precision simulations $[17,23]$. The frequency band models require a high number of narrow bands to resolve rapid variations, causing extreme demands on processing time and memory storage for the simulation. Here, we will discuss and compare different strategies that try to work around these difficulties, while still providing results of high precision.

Most approximate models which are employed to reduce the number of frequency bands are either derived using asymptotic analysis, like in [12] where the so-called frequency averaged $\mathrm{SP}_{n}$ equations are derived, or using fitting techniques combined with approximations, like in the so-called weighted sum of grey gases [17].

Here, we discuss an a posteriori method for automated frequency averaging based on proper orthogonal decomposition (POD) with respect to the frequency variable. This method is widely discussed in literature during the last two decades. The original concept goes back to Pearson [18]. The method is also known as Karhunen-Loève decomposition [8,13] or principal component analysis [7]. It provides an optimally ordered, orthonormal basis in the least-squares sense for a given set of theoretical, experimental or computational data [3]. POD falls into the general category of projection methods where the dynamical system is projected onto a subspace of the original phase space. In combination with Galerkin projection it provides a powerful tool to derive surrogate models for high-dimensional or even infinite dimensional dynamical systems, since the subspace is composed of basis functions inheriting already special characteristics of the overall solution. This is in contrast to standard finite element discretizations where the choice of the basis functions is in general independent of the system dynamics.

This paper is organized as follows. In the remaining part of the introduction, we will present the well-known $\mathrm{SP}_{1}$ equations on which we build our new model reduction method of proper orthogonal decomposition with respect to the frequency variable that is the subject of our paper. In the second section, we focus on $\mathrm{POD}$, deriving it from $\mathrm{SP}_{1}$ band models, discussing its implementation and numerical results. The third section deals with two other model reduction techniques, i.e. the frequency averaged $\mathrm{SP}_{n}$ model and the weighted sum of grey gases. Here, we present the first two dimensional simulations for the former model. Finally, section 4 contains the comparison of all three discussed models and conclusions are given in section 5.

\subsection{The $\mathrm{SP}_{1}$ equations}

The $\mathrm{SP}_{1}$ equations form the basis of our reduced models. Following an overview over the used notation, a short introduction into the frequency-dependent and band formulation of $\mathrm{SP}_{1}$ is given in this subsection; for details, the reader is referred to the introductory sections of [9].

\subsubsection{Notation}

The physical model is described using $t$ for time, $x$ for spatial coordinates; the temperature is denoted by $T$, the radiation intensity by $I$. (For the $\mathrm{SP}_{n}$ models that include the $\mathrm{SP}_{1}$ model as their most basic case, the intensity is replaced by direction-independent radiation fluxes $\phi$ by integrating $I$ over all directions.) The model further depends on the following physical parameters: $\sigma$ is a scattering, $\kappa$ an absorption coefficient; $k_{c}$ denotes the thermal conductivity, $h_{c}$ the convective heat transfer coefficient. $\rho_{m}$ is the density, $c_{m}$ the specific heat capacity. The refractive index of the medium is denoted by $n_{m}$. 


\begin{tabular}{r|l|l} 
Parameter & Value & Description \\
\hline$t_{\text {ref }}$ & $18704 \mathrm{~s}$ & reference time \\
$x_{\text {ref }}$ & $0.1 \mathrm{~m}$ & reference length \\
$T_{\text {ref }}$ & $1 \mathrm{~K}$ & reference temperature \\
$I_{\text {ref }}$ & $5 \frac{\mathrm{W}}{\mathrm{m}^{2}}$ & reference radiation intensity \\
$\kappa_{\text {ref }}$ & $3 \mathrm{~m}^{-1}$ & reference absorption coefficient \\
$k_{c, \text { ref }}$ & $1.672 \frac{\mathrm{W}}{\mathrm{mK}}$ & reference coefficient of thermal conductivity \\
$h_{c, \text { ref }}$ & $5 \frac{\mathrm{W}}{\mathrm{m}^{2} \mathrm{~K}}$ & reference convective heat transfer coefficient \\
$\rho_{m}$ & $2514.8 \frac{\mathrm{kg}}{\mathrm{m}^{3}}$ & density \\
$c_{m}$ & $1239.6 \frac{\mathrm{J}}{\mathrm{kg} \mathrm{K}}$ & specific heat capacity
\end{tabular}

Table 1: Reference values

The equations presented here use non-dimensional variables; the scaling is given by

$$
\begin{gathered}
t^{*}=\frac{t}{t_{\mathrm{ref}}}, \quad x^{*}=\frac{x}{x_{\mathrm{ref}}}, \quad T^{*}=\frac{T}{T_{\mathrm{ref}}}, \quad I^{*}=\frac{I}{I_{\mathrm{ref}}}, \\
\sigma^{*}=\frac{\sigma}{\sigma_{\mathrm{ref}}+\kappa_{\mathrm{ref}}}, \quad \kappa^{*}=\frac{\kappa}{\sigma_{\mathrm{ref}}+\kappa_{\mathrm{ref}}}, \quad k_{c}^{*}=\frac{k_{c}}{k_{c, \mathrm{ref}}}, \quad h_{c}^{*}=\frac{h_{c}}{h_{c, \mathrm{ref}}} .
\end{gathered}
$$

The subscript "ref" is used for the corresponding reference values; these are assumed to fulfill the relations

$$
\begin{gathered}
t_{\text {ref }}=c_{m} \rho_{m}\left(\sigma_{\text {ref }}+\kappa_{\text {ref }}\right) x_{\text {ref }}^{2} \frac{T_{\text {ref }}}{I_{\text {ref }}}, \\
k_{c, \text { ref }}=\frac{I_{\text {ref }}}{\left(\sigma_{\text {ref }}+\kappa_{\text {ref }}\right) T_{\text {ref }}}, \quad h_{c, \text { ref }}=\frac{I_{\text {ref }}}{T_{\text {ref }}} .
\end{gathered}
$$

The parameter $\epsilon$ is a reference opacity and given by

$$
\epsilon=\frac{1}{\left(\sigma_{\mathrm{ref}}+\kappa_{\mathrm{ref}}\right) x_{\mathrm{ref}}} .
$$

In the following, only the scaled values will be used, without denoting them explicitly with the stars. The reference values used in our numerical simulations can be found in table 1. As we assume the absence of scattering in the medium, no $\sigma_{\text {ref }}$ is given, an $\sigma$ and $\sigma^{*}$ are zero.

Let $\Omega$ be a bounded domain, subset of $\mathbb{R}^{d}, d \in\{1,2,3\}$, representing the geometry of the medium, and let $\mathrm{n}$ be the outward normal of $\Omega$ on $\partial \Omega$. Let $\left(0, t_{\text {end }}\right)$ be the time interval used in the simulation, and define $Q$ and $\Sigma$ by

$$
\begin{aligned}
& Q:=\Omega \times\left(0, t_{\text {end }}\right), \\
& \Sigma:=\partial \Omega \times\left(0, t_{\text {end }}\right) .
\end{aligned}
$$




\subsubsection{Frequency-dependent $\mathrm{SP}_{1}$ equations}

The frequency dependent $\mathrm{SP}_{1}$ equations that can be derived as an approximation of the full radiative heat transfer equations under the assumption of an optically thick, diffusive situation [11], are given by

$$
\begin{gathered}
\partial_{t} T-\nabla \cdot\left(k_{c} \nabla T\right)=\int_{\nu_{0}}^{\infty} \nabla \cdot\left(\frac{1}{3(\sigma+\kappa)} \nabla \phi\right) \mathrm{d} \nu \\
\forall \nu>\nu_{0}: \quad-\epsilon^{2} \nabla \cdot\left(\frac{1}{3(\sigma+\kappa)} \nabla \phi\right)+\kappa \phi=4 \pi \kappa B_{\text {glass }}^{*}(T, \nu)
\end{gathered}
$$

in $Q$,

$$
\begin{gathered}
k_{c} \mathbf{n} \cdot \nabla T=\frac{h_{c}}{\epsilon}\left(T_{b}-T\right)+\frac{\alpha \pi}{\epsilon} \int_{0}^{\nu_{0}}\left(B_{\text {air }}^{*}\left(T_{b}, \nu\right)-B_{\text {air }}^{*}(T, \nu)\right) \mathrm{d} \nu \\
\frac{\epsilon}{3(\sigma+\kappa)} \mathbf{n} \cdot \nabla \phi=\frac{1-2 r_{1}}{2+6 r_{2}}\left(4 \pi B_{\text {glass }}^{*}\left(T_{b}, \nu\right)-\phi\right)
\end{gathered}
$$

on $\Sigma$, and

$$
T(x, 0)=T_{0}(x), \quad x \in \Omega
$$

as initial condition.

Here, $r_{1}$ and $r_{2}$ are given as

$$
r_{1}=0.2855742 \quad r_{2}=0.1452082
$$

(see [11]). $B_{m}^{*}$ is given by the scaled black-body radiation intensity at a frequency $\nu$ for a temperature $T \cdot T_{\text {ref }}$

$$
B_{m}^{*}(T, \nu)=\frac{B_{m}\left(T \cdot T_{\mathrm{ref}}, \nu\right)}{I_{\text {ref }}},
$$

where $B_{m}$ is the Planck function describing monochromatic black-body intensity

$$
B_{m}(T, \nu)=\frac{n_{m}^{2}}{c_{0}^{2}} \cdot \frac{2 h_{P} \nu^{3}}{\exp \left(h_{P} \nu /\left(k_{B} T\right)\right)-1} .
$$

In this expression, $h_{P}=6.62608 \cdot 10^{-34} \mathrm{Js}$ is the Planck, $k_{B}=1.38066 \cdot 10^{-23} \mathrm{~J} / \mathrm{K}$ is the Boltzmann constant. $n_{m}$ is the refractive index giving the ratio of the speed of light in vacuum $c_{0}$ and in the medium $c$

$$
n_{m}=\frac{c_{0}}{c} \text {. }
$$

For glass, $n_{\text {glass }}=1.46$ is a valid choice; for the surrounding air we set $n_{\text {air }}=1 . \nu_{0}$ is the frequency up to which the glass is opaque and absorbs radiation; the opacity in the rest of the spectrum is given by $1 / \kappa$, and $\sigma$ is a scattering coefficient.

Remark 1.1. For a mathematical investigation of system (4) we refer to [20], where also an optimal control problem is considered. During the last years this model proved to be a reliable substitute for the full radiative heat transfer problem $[24,23,11]$. 


\subsubsection{Frequency-band $\mathrm{SP}_{1}$ equations}

The frequency band $\mathrm{SP}_{1}$ equations are derived by dividing the frequency space into discrete bands $\left[\nu_{i-1}, \nu_{i}\right], i=1,2, \ldots, N$ and integrating the frequency dependent $\mathrm{SP}_{1}$ equations over these bands using a simple quadrature rule,

$$
\phi_{i}:=\int_{\nu_{i-1}}^{\nu_{i}} \phi \mathrm{d} \nu
$$

i.e. we use a piecewise constant finite element ansatz with respect to the frequency. Under the assumption that $\kappa$ and $\sigma$ are (nearly) constant on the frequency bands

$$
\left.\left.\kappa(\nu)=\kappa_{i}, \quad \sigma(\nu)=\sigma_{i} \quad \text { for } \nu \in\right] \nu_{i-1}, \nu_{i}\right]
$$

we get the $\mathrm{SP}_{1}$ frequency band equations with

$$
\begin{gathered}
\partial_{t} T-\nabla \cdot\left(k_{c} \nabla T\right)=\sum_{i=1}^{N} \nabla \cdot\left(\frac{1}{3\left(\sigma_{i}+\kappa_{i}\right)} \nabla \phi_{i}\right) \\
-\epsilon^{2} \nabla \cdot\left(\frac{1}{3\left(\sigma_{i}+\kappa_{i}\right)} \nabla \phi_{i}\right)+\kappa_{i} \phi_{i}=4 \pi \kappa_{i} \int_{\nu_{i-1}}^{\nu_{i}} B_{\text {glass }}^{*}(T, \nu) \mathrm{d} \nu
\end{gathered}
$$

for $i=1,2, \ldots, N$ in the interior, and

$$
\begin{gathered}
k_{c} \mathbf{n} \cdot \nabla T=\frac{h_{c}}{\epsilon}\left(T_{b}-T\right)+\frac{\alpha \pi}{\epsilon} \int_{0}^{\nu_{0}}\left(B_{\text {air }}^{*}\left(T_{b}, \nu\right)-B_{\text {air }}^{*}(T, \nu)\right) \mathrm{d} \nu \\
\frac{\epsilon}{3\left(\sigma_{i}+\kappa_{i}\right)} \mathbf{n} \cdot \nabla \phi_{i}=\frac{1-2 r_{1}}{2+6 r_{2}}\left(4 \pi \int_{\nu_{i-1}}^{\nu_{i}} B_{\text {glass }}^{*}\left(T_{b}, \nu\right) \mathrm{d} \nu-\phi_{i}\right)
\end{gathered}
$$

for $i=1,2, \ldots, N$ on the boundary, and finally

$$
T(x, 0)=T_{0}(x)
$$

as initial condition.

Remark 1.2. The high number of frequency bands required in applications cause the above system to be of significant size. One often encounters up to 300 frequency bands, i.e. one has to solve one nonlinear parabolic PDE coupled with 300 elliptic equations. For $\mathrm{SP}_{n}$ models with $n$ higher than 1 , this problem will be even worse, as new flux variables are needed for each radiation band [11].

\section{POD and basis-transformation of the $\mathrm{SP}_{1}$ equations}

After discussing $\mathrm{SP}_{1}$ in its frequency-dependent and band variant, we will now focus on a basistransformed band variant of $\mathrm{SP}_{1}$, which will, in combination with proper orthogonal decomposition (POD), finally lead to the new POD frequency averaged model. The presentation of the POD equations for $\mathrm{SP}_{1}$ will be followed by details concerning our implementation and the numerical results that were obtained. 


\subsection{Basis-transformed frequency-band $\mathrm{SP}_{1}$ equations}

In section 1.1.3, frequency bands were chosen so that the absorptivity of the medium was almost constant over each band. For realistic spectral data with large variations of the absorption coefficient, this approach leads to an undesirably high number of required bands and thus to a high number of flux variables; therefore it is important to develop a variant of the frequency band $\mathrm{SP}_{1}$ model that allows to reduce the number of flux variables by representing the full spectrum using fewer coordinates.

This is done by setting

$$
\phi_{i}:=\sum_{j=1}^{M} m_{i j} \psi_{j},
$$

where $M \leq N$, in most cases $M \ll N$, thus representing the "natural bands" $\phi_{i}$ by "artificial bands" $\psi_{j}$. One possibility to find the $m_{i j}$ is the application of proper orthogonal decomposition to discover the most important frequency modes; this approach will be discussed in detail in the next section; meanwhile, $m_{i j}$ will be treated as given data. However, we will assume that the matrix $P:=\left(m_{i j}\right)_{i, j}$ is orthonormal; this allows for simpler notation, as $P^{-1}=P^{T}$ and the matrix $P^{T} \cdot P$ that will appear in the flux equations in $\Omega$ will be just the identity.

Applying the basis transformation to the frequency band $\mathrm{SP}_{1}$ equations of the last chapter, we get

$$
\begin{aligned}
\partial_{t} T-\nabla \cdot\left(k_{c} \nabla T\right) & =\sum_{j=1}^{M} \nabla \cdot\left(\sum_{i=1}^{N} \frac{m_{i j}}{3\left(\sigma_{i}+\kappa_{i}\right)} \nabla \psi_{j}\right) \\
-\sum_{k=1}^{M} \epsilon^{2} \nabla \cdot\left(\sum_{i=1}^{N} \frac{m_{i j} m_{i k}}{3 \kappa_{i}\left(\sigma_{i}+\kappa_{i}\right)} \nabla \psi_{k}\right)+\psi_{j} & =4 \pi \sum_{i=1}^{N} m_{i j} \int_{\nu_{i-1}}^{\nu_{i}} B_{\text {glass }}^{*}(T, \nu) \mathrm{d} \nu
\end{aligned}
$$

in $Q$ and

$$
\begin{gathered}
k_{c} \mathbf{n} \cdot \nabla T=\frac{h_{c}}{\epsilon}\left(T_{\mathrm{b}}-T\right)+\frac{\alpha \pi}{\epsilon} \int_{0}^{\nu_{0}}\left(B_{\text {air }}^{*}\left(T_{\mathrm{b}}, \nu\right)-B_{\text {air }}^{*}(T, \nu)\right) \mathrm{d} \nu \\
\sum_{k=1}^{M} \epsilon \sum_{i=1}^{N} \frac{m_{i j} m_{i k}}{3 \kappa_{i}\left(\sigma_{i}+\kappa_{i}\right)} \mathbf{n} \cdot \nabla \psi_{k}= \\
\quad \frac{1-2 r_{1}}{2+6 r_{2}}\left(4 \pi \sum_{i=1}^{N} \frac{m_{i j}}{\kappa_{i}} \int_{\nu_{i-1}}^{\nu_{i}} B_{\text {glass }}^{*}\left(T_{\mathrm{b}}, \nu\right) \mathrm{d} \nu-\sum_{k=1}^{M} \sum_{i=1}^{N} \frac{m_{i j} m_{i k}}{\kappa_{i}} \psi_{k}\right)
\end{gathered}
$$

on $\Sigma$.

As one can see from these equations, all summations over $i \in\{1, \ldots, N\}$ can be done in advance, giving the vectors

$$
\begin{gathered}
A_{1}:=\left(\sum_{i=1}^{N} \frac{m_{i j}}{\left(\sigma_{i}+\kappa_{i}\right)}\right)_{j}=P^{T} \cdot\left(\frac{1}{\left(\sigma_{i}+\kappa_{i}\right)}\right)_{i=1}^{N} \\
A_{2}:=\left(\sum_{i=1}^{N} \frac{m_{i j}}{\kappa_{i}}\right)_{j}=P^{T} \cdot\left(\frac{1}{\kappa_{i}}\right)_{i=1}^{N}
\end{gathered}
$$


(where $A_{1}=A_{2}=: A$ when scattering is neglected) and the matrices

$$
\begin{gathered}
B:=\left(\sum_{i=1}^{N} \frac{m_{i j} m_{i k}}{\kappa_{i}}\right)_{j, k}=P^{T} \cdot\left(\left(\frac{1}{\kappa_{i}}\right)_{i=1, j=1}^{i=N, j=M} \otimes P\right) \\
G:=\left(\sum_{i=1}^{N} \frac{m_{i j} m_{i k}}{\kappa_{i}\left(\sigma_{i}+\kappa_{i}\right)}\right)_{j, k}=P^{T} \cdot\left(\left(\frac{1}{\kappa_{i}\left(\sigma_{i}+\kappa_{i}\right)}\right)_{i=1, j=1}^{i=N, j=M} \otimes P\right),
\end{gathered}
$$

with the matrix $P$ defined as $P=\left(m_{i j}\right)_{i, j}, i \in\{1, \ldots, N\}, j \in\{1, \ldots, M\}$ (for POD, $P$ is the POD basis matrix) and $\otimes$ being the element-wise matrix product. The matrices

$$
\left(\frac{1}{\kappa_{i}\left(\sigma_{i}+\kappa_{i}\right)}\right)_{i=1, j=1}^{i=N, j=M} \text { and }\left(\frac{1}{\kappa_{i}}\right)_{i=1, j=1}^{i=N, j=M}
$$

are the concatenation of the $N$-column-vectors $\left(\kappa_{i}^{-1}\left(\sigma_{i}+\kappa_{i}\right)^{-1}\right)_{i}$ and $\left(\kappa_{i}^{-1}\right)_{i}$, respectively to a matrix of $N$ rows and $M$ columns.

Remark 2.1. Assuming that absorption and scattering are independent of space and due to the special structure of the matrices $B$ and $G$ given above, one can apply diagonalization techniques to convert these (full) matrices simultaneously to diagonal matrices and increase the performance of the algorithm even more. This is what we call diagonalized POD. In addition to being more efficient, diagonalized POD produces frequency bands that do not couple and can thus be interpreted as a generalization of band-models (although the frequency modes are linear independent, they overlap strongly, what is not the case for conventional frequency band models).

\subsection{Computation of an optimal frequency basis using POD}

In the discussion of the basis-transformed $\mathrm{SP}_{1}$ variant above, we left open the details of how to find a suitable basis. Now we use proper orthogonal decomposition with respect to the frequency variable that will yield an optimal result in the least-squares sense.

The problem that has to be dealt with in our context is the question whether it is possible to express the (discrete) spectra $F_{1}:=\left(\phi_{i}\right)_{i=1}^{N}$ that are encountered in all grid points of the discretization of $\Omega \times\left(0, t_{\text {end }}\right)$ in time and space using a vector $F_{2}:=\left(\psi_{j}\right)_{j=1}^{M}$ of flux variables with a dimension $M$ considerably smaller than the number $N$ of frequency bands. In the ideal case, the representation $F_{1}=P \cdot F_{2}$ should be exact; as this is not possible in general, we demand that the approximation error $\left\|F_{1}-P \cdot F_{2}\right\|$ in a suitable norm should be minimized for given dimensions $N$ and $M$.

This problem is solved by proper orthogonal decomposition [10,6], which is an a posteriori method to compute this optimal basis. However, being a data based method, one solution of the original problem is necessary in order to compute the suitable basis transform. This is not as bad as it might sound, as the basis computed from this initial dataset can be used for a broad range of similar problems, what is especially important when thinking of applying this model reduction technique in the context of optimal control.

The initial solution of the full problem yields via the method of snapshots [25] spectral data $\tilde{S}=$ $\left(F_{1, i}\right)_{i}, i \in I$, for each grid point in space and time of the discretization. As further processing consists of algorithms that are computationally expensive for large size of $I$, the complete set of spectral information is replaced by a suitable subset $S=\left(F_{1, j}\right)_{j}, j \in J \subset I$, that is still representative 
for the whole, such that one still gets the correct dynamics of the system. In order to find a small basis of a subspace of the span of all $F_{1, i}, i \in I$, that allows the approximate representation of all $F_{1, i}$ up to high accuracy, we build the correlation matrix $C$ given by

$$
C=S^{T} \cdot S
$$

using the scalar product of $\mathbb{R}^{N}$. $C$ is positive semidefinite; all eigenvalues $d_{i}$ of $C$ are therefore real and nonnegative. Using appropriate numerical algorithms, the eigenvectors $v_{i}$ (sorted by decreasing eigenvalue $d_{i}$ ) can be computed, combined into a matrix $V$, and the frequency eigenmodes matrix $E$ is given by

$$
E=S \cdot V
$$

As we demanded in 2.1 that the POD basis $P$ should be orthonormal, this step is followed by an orthonormalization of the first $M$ columns of $E$, yielding the POD basis $P$. From this matrix and the opacity dataset, the vector $A$ and the matrices $B$ and $G$ can be computed. After optional diagonalization of $B$ and $G$ (and corresponding updates to $P$ and $A$ ) for diagonalized POD, the POD dataset is complete.

Remark 2.2. It can be shown that the POD basis vectors are ordered in a way that the approximation of the spectral snapshots using the first $k$ basis vectors is the best approximation that can be obtained using an arbitrary basis of $k$ vectors [10].

Still, one has to decide how many basis vectors will be selected for the reduced spectral model. In terms of a dynamical system, large eigenvalues correspond to main characteristics of the system, while small eigenvalues give only small perturbations of the overall dynamics. The goal is to choose $\ell$ small enough while the relative information content [1] of the basis defined by

$$
I(\ell):=\frac{\sum_{k=1}^{\ell} d_{k}}{\sum_{k=1}^{N} d_{k}}
$$

is near to one. Typically, the magnitude of the eigenvalues decreases very rapidly for the first values, so that numbers of eight, five and sometimes even less eigenvectors proved to be enough for simulations with satisfying accuracy; this will also be seen in the presentation of the computed eigenmodes in 2.4.

The algorithm used to generate the POD parameter set is given below.

Algorithm 2.3. Algorithm for computing the POD coefficient dataset

\section{begin}

- let $m$ be the number of desired POD bands

- load simulation dataset and extract samples

- optional: compute time derivatives of simulated data and add samples to the set of samples from the previous step

- form the sample matrix $S$ with the samples as columns

- compute correlation $C$ matrix as $C:=S^{T} \cdot S$

- compute eigenvectors $v_{i}$ and eigenvalues $d_{i}$ of correlation matrix $C$, sorted so that $d_{i}>d_{i+1}$

- form the matrix $V$ with the $v_{i}$ as its rows

- compute the full frequency eigenmode matrix $\tilde{E}$ as $\tilde{E}:=S \cdot V$

- select the first $m$ columns of $\tilde{E}$ into the eigenmode matrix $E: E=\tilde{E}(:, 1: m)$

- optional: normalize the columns of $E$ so that they all have norm 1 
- perform $Q R$ factorization on $E: Q \cdot R=E$

- store the first $m$ columns of $Q$ as the POD basis $P:=Q(:, 1: m)$, set

$\circ k_{1}$ as the column vector of values $1 / \kappa_{i}$

$\circ K_{1}$ as the column vector of values $1 / \kappa_{i}$, repeated into a matrix of $m$ columns

$\circ K_{2}$ as the column vector of values $1 / \kappa_{i}^{2}$, repeated into a matrix of $m$ columns

- and compute

$\circ A:=P^{T} \cdot k_{1}$

$\circ B:=P^{T} \cdot\left(K_{1} \otimes P\right)$

$\circ G:=P^{T} \cdot\left(K_{2} \otimes P\right)$

- save the matrices $A, B, G$ and $P$ as the POD parameter set

end

\subsection{Implementation and Numerical Results}

Now we present numerical results and compare them to two other reference models. The physical parameters used for all simulations are given in table 2. Due to the choice of the scaling coefficients, the scaled values $k_{c}^{*}$ and $h_{c}^{*}$ were both identical to 1 . The frequency dependent absorption coefficients used are given in figure 1.

\begin{tabular}{r|l|l} 
Parameter & Value & Description \\
\hline$k_{c}$ & $1.672 \frac{\mathrm{W}}{\mathrm{mK}}$ & $\begin{array}{l}\text { coefficient of thermal conductivity } \\
\text { convective heat transfer coefficient }\end{array}$ \\
$h_{c}$ & $5 \frac{\mathrm{W}}{\mathrm{m}^{2} \mathrm{~K}}$ &
\end{tabular}

Table 2: Physical properties

The geometry was the square $[-1,1] \times[-1,1]$ in scaled coordinates, corresponding to an edge length of $0.2 \mathrm{~m}$. The material was cooled in the scaled time interval $[0,0.1]$, corresponding to approximately thirty minutes of cooling time; the boundary temperature was decreased linearly from an initial temperature of $1000 \mathrm{~K}$ (that was also the initial temperature of the glass) to $400 \mathrm{~K}$. For simulations that show the good suitability of the POD dataset generated for this cooling scenario, the initial temperature was modified within the values of $800 \mathrm{~K}, 900 \mathrm{~K}, 1100 \mathrm{~K}$, and $1200 \mathrm{~K}$.

In order to create easily comparable results, all simulations (for the full and several reduced models) were based on identical numerical settings. The spatial domain was discretized using a $25 \times 25$ grid. The spatial discretization of the differential equations was accomplished using standard linear finite elements. The time interval was discretized using an equidistant grid of 1250 intervals. The time discretization was done using a semiimplicit scheme based on a modified implicit Euler's method. The semiimplicit approach also simplified the implementation of the highly nonlinear $\mathrm{GSP}_{2}$ model (discussed in 3.2).

Remark 2.4. For the spatial and temporal discretization described above, a model consisting of 283 frequency bands yields a total of $25 \times 25 \times 1250 \times 284 \approx 2 \cdot 10^{8}$ degrees of freedom. A finer grid, higher spatial dimension or the use of an $\mathrm{SP}_{n}$ model with $n>2$, which could be desirable in practical use, even worsens the size of the problem. These numbers show that some sort of model reduction is unavoidable for solving real life problems (especially when optimization problems are considered). 


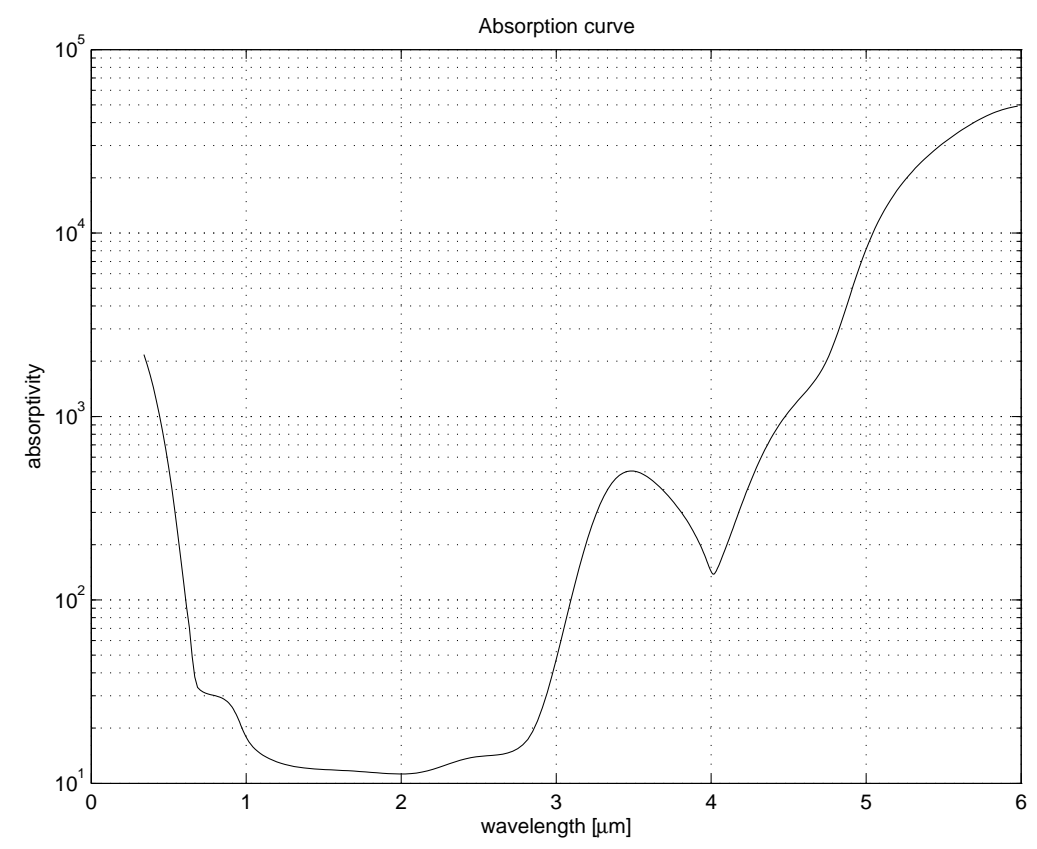

Figure 1: Absorption curve

\subsection{Computed frequency eigenmodes}

As outlined in 2.2, computing POD bands consists of taking spectral snapshots from a simulation using the full model, computing the eigenvalues and eigenvectors of the correlation matrix of these snapshots, and selecting eigenvectors with the highest eigenvalues to compute the POD bands. For the snapshots, every 15 th temporal and every seventh spatial discretization point was selected. Based on a simulation using the full model, POD datasets for 1, 2, 3, 4, 6, 8 and 10 artificial POD bands were created. The information content of the first ten eigenmodes computed from the full model snapshots are given in table 3. The third column contains the cumulative relative information content of all modes up to the given index, as difference from total $100 \%$. As one can clearly see, the first mode dominates all others.

Remark 2.5. Using diagonalized POD, the results obtained after diagonalization can be interpreted as linear independent frequency eigenmodes of the spectrum with corresponding opacities. Due to the diagonalization process, these frequency eigenmodes do not couple, as is the case for more conventional frequency band models; however, diagonalized POD produces strongly overlapping "bands", so that they should be called "modes" to avoid confusion.

Results from proper orthogonal decomposition with $k$ and $k+1$ bands may have completely different frequency eigenmodes, but $k$ common opacity values; for each new POD band, a new opacity is added, but in general no modes are preserved. Figure 2 show the frequency eigenmodes computed for POD band models consisting of 1, 3, 6 and 8 bands. Table 4 shows the opacities computed for the POD band models, sorted by the count of bands of the model they first appear in (which indicates the importance of the opacity), as specified in the third column. 


\begin{tabular}{r|ll} 
mode \# & rel. inform. content $(\%)$ & cum. rel. inform. content $(\%)$ \\
\hline 1 & 99.162266 & $100-0.837733$ \\
2 & 0.764715 & $100-0.073017$ \\
3 & 0.063888 & $100-0.009129$ \\
4 & 0.007681 & $100-0.001448$ \\
5 & 0.001036 & $100-4.116644 \cdot 10^{-04}$ \\
6 & $2.971880 \cdot 10^{-04}$ & $100-1.144763 \cdot 10^{-04}$ \\
7 & $6.439107 \cdot 10^{-05}$ & $100-5.008525 \cdot 10^{-05}$ \\
8 & $2.883304 \cdot 10^{-05}$ & $100-2.125220 \cdot 10^{-05}$ \\
9 & $9.837686 \cdot 10^{-06}$ & $100-1.141452 \cdot 10^{-05}$ \\
10 & $7.299206 \cdot 10^{-06}$ & $100-4.115314 \cdot 10^{-06}$
\end{tabular}

Table 3: Information content of POD modes

\begin{tabular}{rrr}
$i$ & \multicolumn{1}{c}{$\kappa_{i}$} & first appearance \\
\hline 1 & 7.2938 & 1 band model \\
2 & 5.2891 & 2 band model \\
3 & 12.9749 & 3 band model \\
4 & 6.3860 & 4 band model \\
5 & 6.4246 & 6 band model \\
6 & 6.9165 & 6 band model \\
7 & 7.9478 & 8 band model \\
8 & 25.3244 & 8 band model \\
9 & 6.2844 & 10 band model \\
10 & 13.4144 & 10 band model
\end{tabular}

Table 4: Opacities of the POD models

\subsection{Simulation results}

The primary goal for the POD model reduction technique is to provide a efficient method for highquality approximation of the full model. The following figures show the approximation error of POD with different numbers of bands; in the two plots in figure 3, the evolution of the mean and maximum error over time is shown, while the plots in figure 4 show the spatial distribution of the approximation error for the last time step. It should be observed that 8 band POD yields a worse approximation than 6 band POD, while 10 band POD is again better than 6 band POD. This can be attributed to the fact that POD finds a best approximating subspace, but not the best approximation for the system dynamics. But there are recent results which allow to account also for this effect [16, 15, 22].

Remark 2.6. From the data presented, it is evident that the POD approximation is worst near the boundary for low number of bands in the reduced model. One reason for this effect is the presence of boundary layers. In order to show that POD results can be enhanced without the need for more complex reduced models, we modified the POD method like proposed in [6]. We increased the dataset used in the proper orthogonal decomposition step by temporal derivatives of the data used so far; this gives higher priority to faster varying modes, i.e. the boundary layers. The plots in figure 5 show comparisons between the original 3 band POD results and the new variant. It can be seen that both 

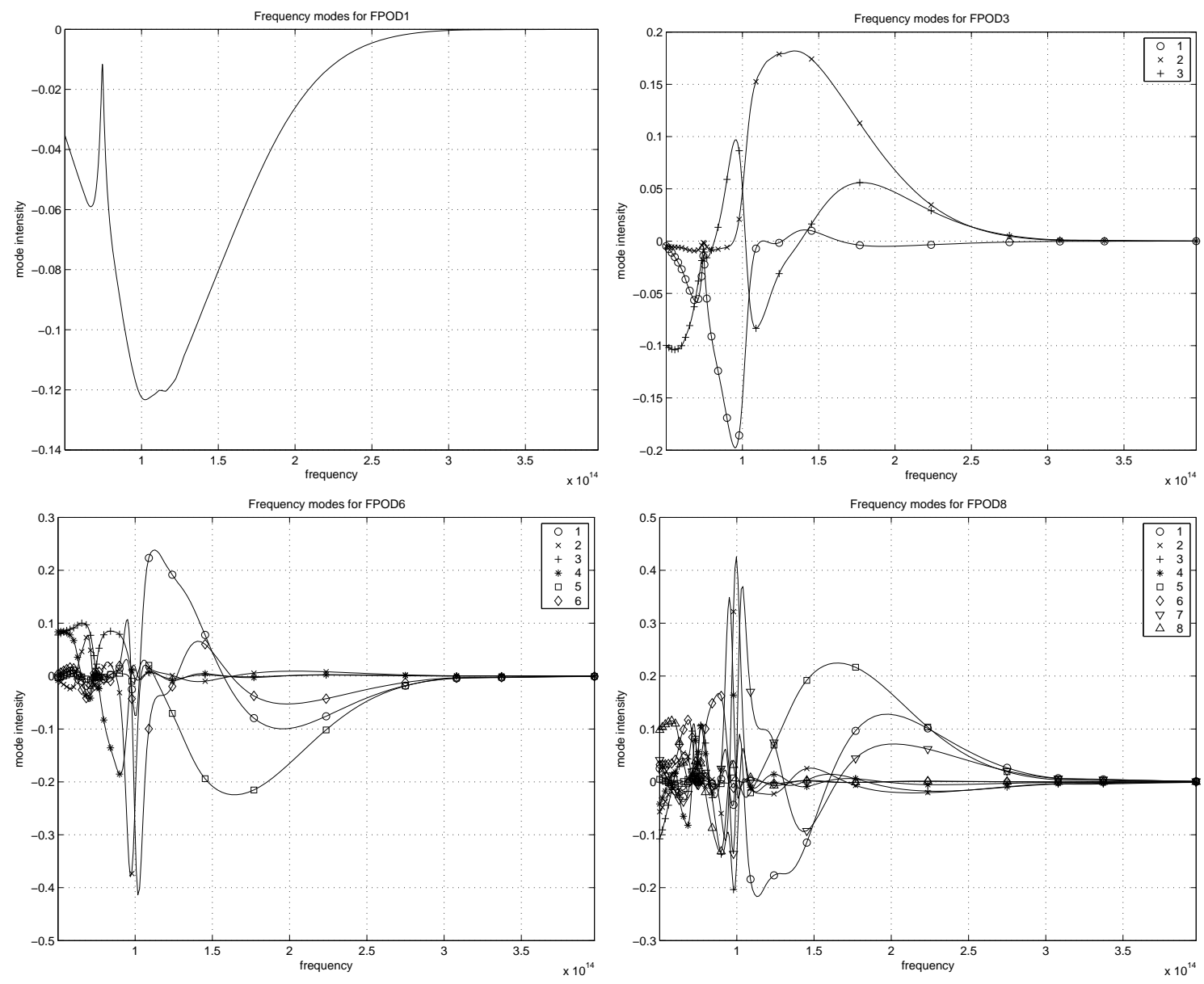

Figure 2: POD frequency modes

maximum and mean error could be reduced noticeably.

\subsubsection{Dependency of the approximation quality on the cooling scenario}

Being an a posteriori method, POD requires a solution of the full system in order to compute the POD coefficients. As the full model has extreme demands on storage and computation time, it is important for the applicability of POD in real-world problems to know about the sensitivity of the approximation quality with respect to variations in the cooling scenario. Optimization problems, for example, change the boundary temperature function $T_{b}$ in each step of the optimization.

Fortunately, we were able to show that POD gives excellent approximations even for modification of the initial temperature (of the medium and the oven) by $\pm 200 \mathrm{~K}$. The mean and maximum errors for 4 and 10 band POD in simulations using the modified initial temperatures are shown in figure 6. Evidently, the dependency on the cooling profile is only marginal, and the POD datasets computed for a cooling from $1000 \mathrm{~K}$ to $400 \mathrm{~K}$ can be used over a wide range of modified profiles. In the case of 4 band POD, the approximation error decreases with decreasing initial temperature, even below the error for the profile the POD dataset was initially generated for. For 10 band POD, the result is 

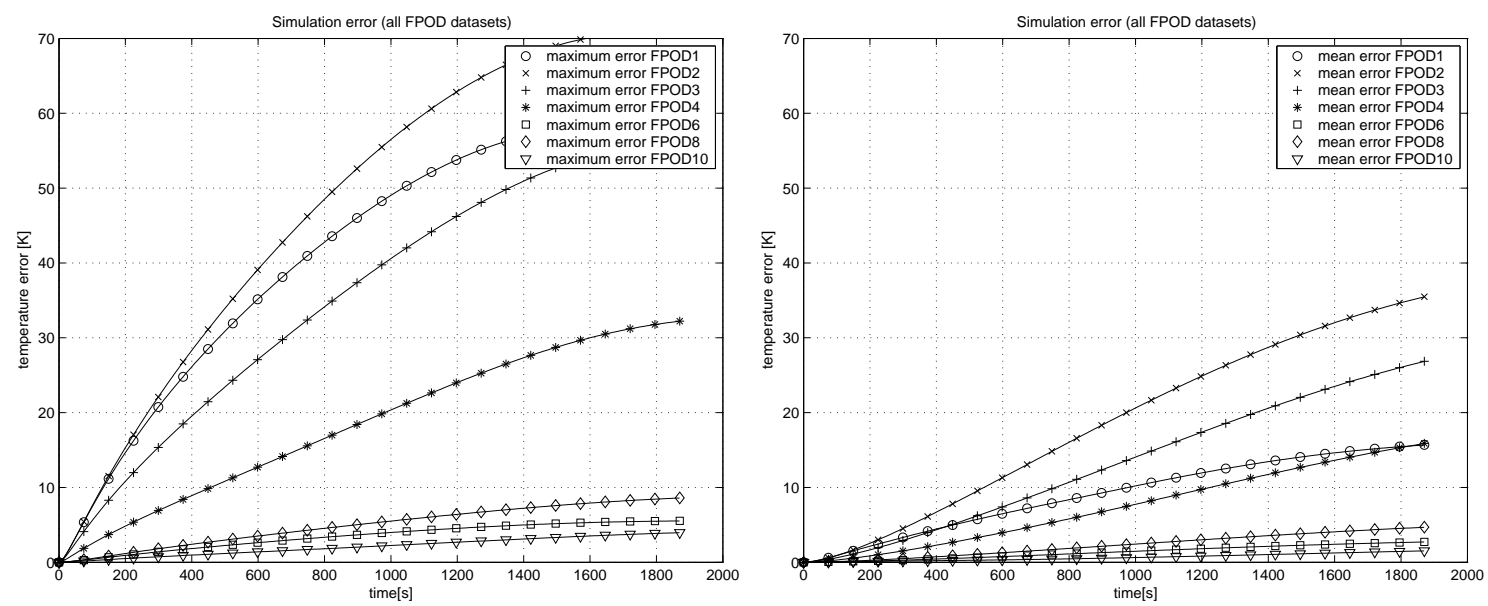

Figure 3: Evolution of the error for POD model

similar, except for an anomaly in the mean error for initial temperatures above $1000 \mathrm{~K}$, which shows a different temporal evolution than for the other cooling profiles.

\section{Other model reduction techniques}

In this section, we will shortly present two other well-known methods for reducing the dimensionality of the discretization in the frequency domains and compare them with the new method we proposed above. The first method, known as weighted sum of grey gases, is based on a physical interpretation of the problem and tries to fit certain parameters to match physical properties of the cooling medium [17]. The second model is derived from asymptotical analysis of the $\mathrm{SP}_{2}$ equations. Model reduction is performed by analytic integration over the frequency domain, yielding a single-band model with opacities that are dependent on temperature [12].

\subsection{Weighted Sum of Gray Gases (WSGG)}

The WSGG model tries to approximate the full model by substituting the medium with a number of gray media (known as "gray gases" because WSGG was first implemented for gaseous media). The opacities and fractions of these gray media are found by solving a fitting problem for the absorptivity of the medium, a physical property that will be introduced below.

The total absorptivity and emissivity of a homogeneous, isothermal medium at temperature $T$ is given by

$$
\alpha(T, s)=\epsilon(T, s)=\frac{1}{I_{b, \text { tot }}(T)} \int_{0}^{\infty}(1-\exp (-\kappa(\nu) s)) I_{b}(\nu, T) \mathrm{d} \nu,
$$

where $I_{b}(\nu, T)$ designates the Planck radiation density at frequency $\nu$ for a black body at temperature $T$, and $I_{b, \text { tot }}$ is the integral of $I_{b}$ over the whole spectrum.

The model parameters of the weighted sum of gray gases model are the weighting factors for the linear combination of the results for the gray gases and the absorption coefficients of these gray gases. These 

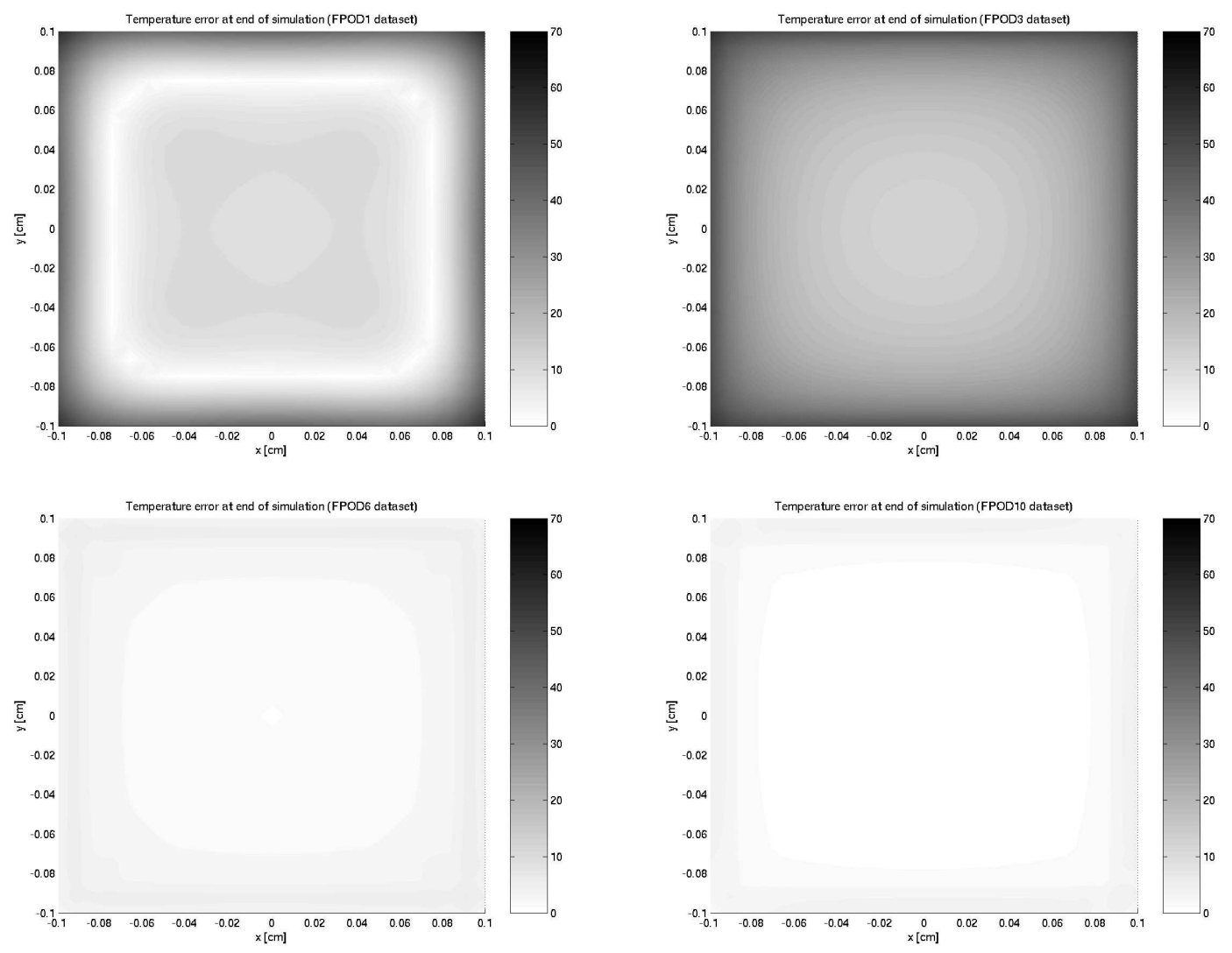

Figure 4: Spatial distribution of the error in last time step, POD model

parameters are found by fitting the total emissivity on a line of characteristic length in the medium with the total emissivity of the linear combination of the gray gases; this yields

$$
\frac{1}{I_{b, \text { tot }}(T)} \int_{0}^{\infty}\left(1-\exp (-\kappa(\nu) s) I_{b}(\nu, T) \mathrm{d} \nu \approx \sum_{k=0}^{K}\left(1-\exp \left(\kappa_{k} s\right)\right) \alpha_{k}(T),\right.
$$

where $\nu$ is the frequency, $\kappa(\nu)$ is the frequency-dependent absorptivity of the real material, $\kappa_{k}$ is the absorptivity of the $k$-th gray gas, $T$ is the temperature and $s$ is a length-parameter. The $\alpha_{k}$ are the weighting factors and may depend on the temperature of the medium, whereas the $\kappa_{k}$ are assumed to be temperature-independent. In order to find appropriate values for $\alpha_{k}$ and $\kappa_{k}$, a (highly nonlinear) least-squares fit is done using a set of temperatures $T_{n}, n=1, \ldots, \# T$, and a set of path length parameters $s_{n}, n=1, \ldots, \#$, , suited to the problem.

Remark 3.1. As we just outlined, the coefficients of WSGG models are found by a nonlinear least squares fit; being an a priori method, the fit requires no data from a previous full-model simulation, as was the case for the POD method, and so far, WSGG seems to be significantly easier in its application. However, the choice of the parameters pathlength $s_{n}, n=1 \ldots, \# s$, and temperature $T_{n}, n=$ $1, \ldots, \# T$, that is used is crucial for the quality of the fit, and without any knowledge of the problem geometry, macroscopic properties of the radiation field and temperatures encountered in the cooling process, it is not clear how to choose these parameter appropriately. The advantage of an a priori 

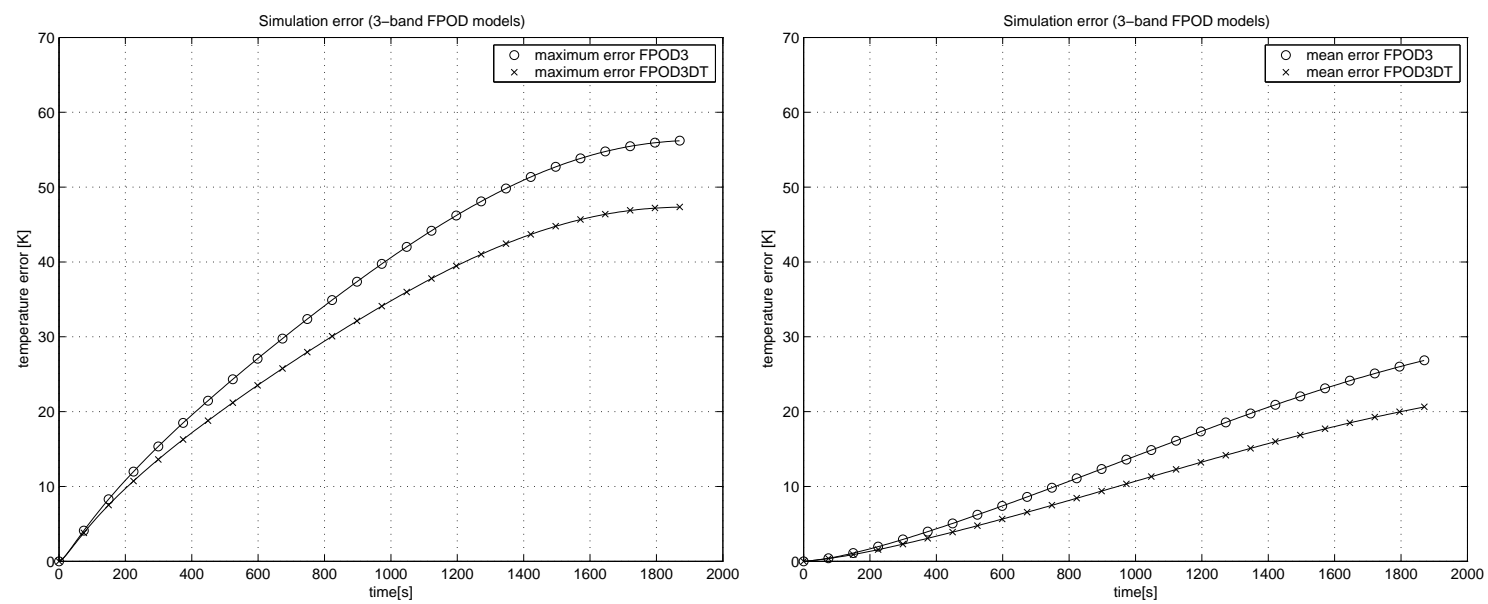

Figure 5: Evolution of the approximation error for enhanced methods
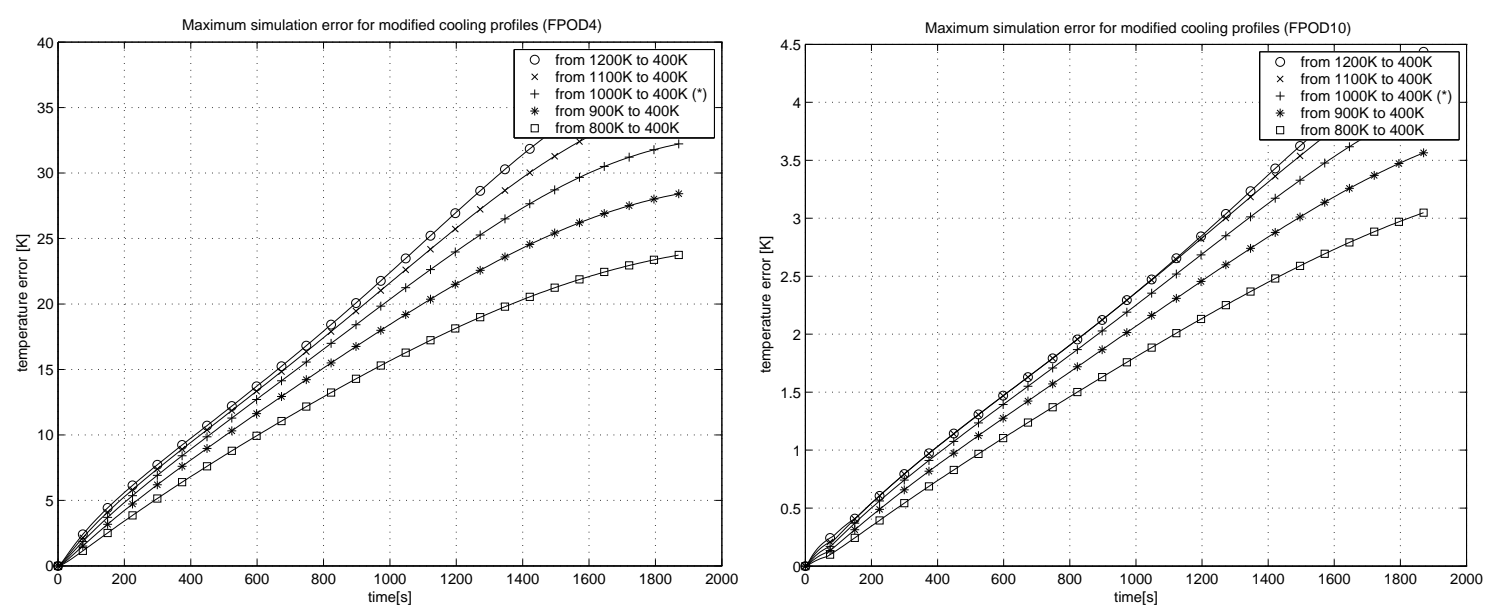

Figure 6: Dependency of approximation error on cooling scenario

method is turned into a disadvantage, because one has to resort to heuristic strategies in order to get WSGG coefficients that lead to good approximation of cooling behaviour.

Four datasets for ten gray gases each were computed, varying the optical pathlengths used in the nonlinear fit, as given in table 5. The grid consisted of approximately 1000 equally distributed grid points for the first three fits, approximately 500 points for the last fit. All fits were computed over the temperature range from $550 \mathrm{~K}$ to $1000 \mathrm{~K}$. The fitting points were equally distributed, using a grid size of $50 \mathrm{~K}$ (the size of the optimization problem depends on the temperature grid size; no finer temperature grid was chosen to keep the computational efforts to a reasonable level of 110 variables).

Relatively good fit results were only obtained using the last two datasets, indicating that the optical pathlengths used in the first two fits were too small. The last dataset gives the best results.

For each of the four datasets simulations were run and results compared to the solution of the full system. The results are given in figure 7, given as evolution of error in time and spatial distribution of 


\begin{tabular}{r|lll} 
dataset \# & pathlength interval $[\mathrm{m}]$ & grid size $[\mathrm{m}]$ & \# of grid points \\
\hline 1 & {$[0.0001,0.01]$} & 0.00001 & 991 \\
2 & {$[0.002,0.2]$} & 0.0002 & 991 \\
3 & {$[0.002,1]$} & 0.001 & 999 \\
4 & {$[0.01,0.5]$} & 0.001 & 491
\end{tabular}

Table 5: WSGG fit parameters
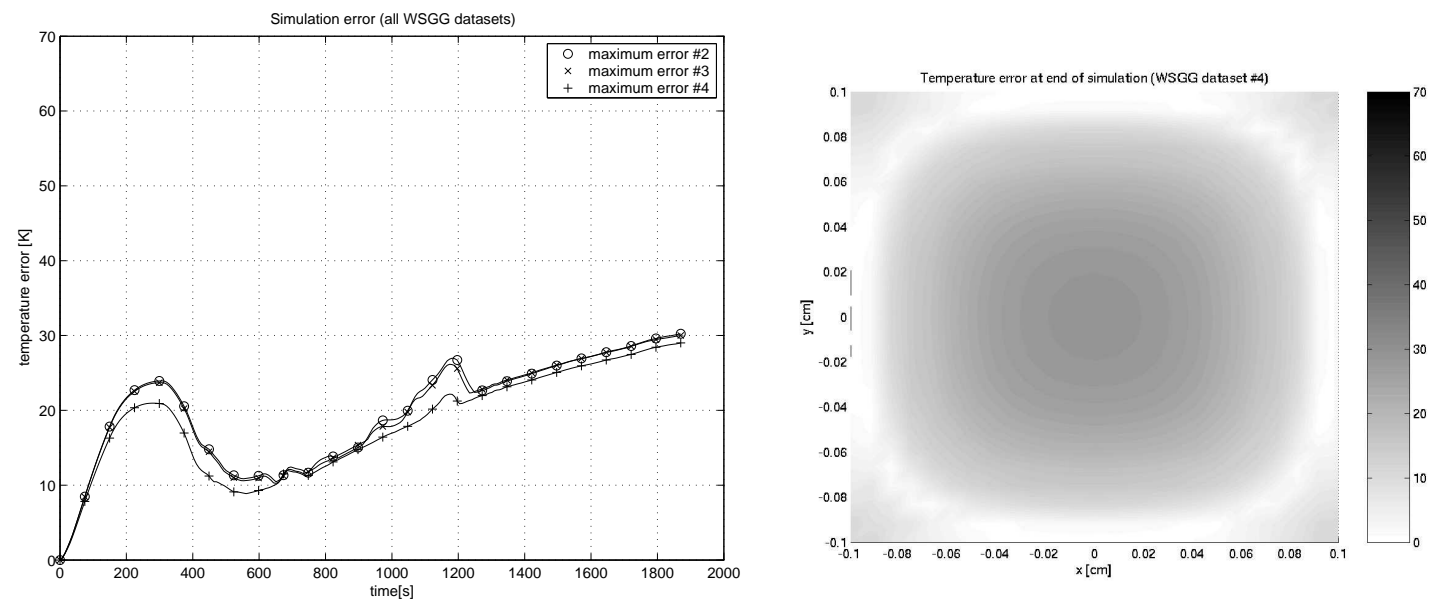

Figure 7: Approximation error for WSGG model

error at the last time step. Note that no results are available for the first dataset, as the corresponding simulation failed to converge. The error graphs show similar behaviour, the mean error increasing over time, with the fourth dataset giving the best results, although the fit was done over a subset of the fit points for the third dataset.

\subsection{Frequency averaged $\mathrm{SP}_{n}$-equations}

Another possibility to derive reduced models in the frequency domain is by integrating the frequency dependent fluxes with respect to the frequency $\nu$ analytically, thus defining a new state variable and producing a frequency averaged single band model. This is done by the $\mathrm{GSP}_{2}$ model discussed in [12], where the following equations are derived for homogeneous media. Given the auxiliary functions

$$
f_{n}(T):=\frac{4 \pi}{n+2} \int_{\nu_{1}}^{\infty} \frac{B(\nu, T)}{\kappa^{n}(\nu)} \mathrm{d} \nu
$$

for $n=1,2,3$ (not correlated to the $n$ in $\mathrm{GSP}_{n}$ ) and variables

$$
\begin{aligned}
\alpha_{1} & :=\frac{4}{5} \cdot \frac{1+3 r_{2}}{1-4 r_{3}} \\
\alpha_{2} & :=\frac{6}{5} \cdot \frac{1-2 r_{1}}{1-4 r_{3}},
\end{aligned}
$$


where the parameters $r_{1}, r_{2}$ and $r_{3}$ are moments of the reflectivity of the medium that depend on $n_{\text {glass }}$ and are, in our case for $n_{\text {glass }}:=1.46$, given as

$$
\begin{aligned}
& r_{1}:=0.2855741980 \\
& r_{2}:=0.1452081942 \\
& r_{3}:=0.08373343569,
\end{aligned}
$$

the equations in $Q$ are given by

$$
\begin{gathered}
\frac{\partial T}{\partial t}=\nabla \cdot(k \nabla T)+\nabla^{2} W \\
-\epsilon^{2} \nabla \cdot\left(\frac{f_{3}^{\prime}(T)}{f_{1}^{\prime}(T)} \nabla W\right)+W=f_{1}(T),
\end{gathered}
$$

whereas the boundary conditions on $\Sigma$ are

$$
\begin{gathered}
\epsilon k \mathrm{n} \cdot \nabla T=h\left(T_{b}-T\right)+\alpha \pi \int_{0}^{\nu_{1}}\left[B^{(a i r)}\left(\nu, T_{b}\right)-B^{(a i r)}(\nu, T)\right] \mathrm{d} \nu, \\
W+\left(\frac{4 \alpha_{1} \epsilon}{3} \cdot \frac{f_{2}^{\prime}(T)}{f_{1}^{\prime}(T)}\right) \mathrm{n} \cdot \nabla W=f_{1}(T)+\alpha_{2}\left[f_{1}\left(T_{b}\right)-f_{1}(T)\right] .
\end{gathered}
$$

The initial condition of the differential-algebraic parabolic system is given as usual by

$$
T(x, 0)=T_{0}(x) .
$$

In this notation, $P^{(a i r)}$ means that the corresponding Planckian has to be computed using the refractive index of air (that is, 1 ) instead of glass ( $n_{\text {glass }}$ ). In this $\mathrm{GSP}_{2}$ model, the new variable $W$ is defined as

$$
W(x, t):=\frac{1}{3} \int_{\nu_{1}}^{\infty} \frac{\phi(x, \nu, t)}{\kappa(\nu)} \mathrm{d} \nu
$$

thus, for space-independent $\kappa, W$ is a absorptivity-scaled flux.

\subsubsection{Implementation and numerical results}

The $\mathrm{GSP}_{2}$ equations can be rewritten substituting the functions $f_{n}, n \in\{1,2,3\}$, to take a form that allows for easier comparison with the $\mathrm{SP}_{n}$ equations. The equations on the domain $\Omega$ are then given by

$$
\begin{gathered}
\frac{\partial T}{\partial t}=\nabla(k \nabla T)+\sum_{i \in I} \nabla^{2} W_{i} \\
-\epsilon^{2} \beta \nabla\left(\frac{\sum_{j \in J(i)} \frac{P_{j}^{\prime}}{\kappa_{j}^{3}}}{\sum_{j \in J(i)} \frac{P_{j}^{\prime}}{\kappa_{j}}} \nabla W_{i}\right)+W_{i}=\frac{4 \pi}{3} \sum_{j \in J(i)} \frac{P_{j}}{\kappa_{j}} \quad \forall i \in I,
\end{gathered}
$$

whereas the boundary conditions are

$$
\begin{gathered}
k \mathrm{n} \cdot \nabla T=\frac{h}{\epsilon}\left(T_{b}-T\right)+\frac{\alpha \pi}{\epsilon}\left(P_{0, b}^{(a)}-P_{0}^{(a)}\right) \\
W_{i}+\gamma \epsilon \frac{\sum_{j \in J(i)} \frac{P_{j}^{\prime}}{\kappa_{j}^{2}}}{\sum_{j \in J(i)} \frac{P_{j}^{\prime}}{\kappa_{j}}} \mathrm{n} \cdot \nabla W_{i}=\frac{4 \pi}{3} \delta_{i} \quad \forall i \in I .
\end{gathered}
$$


In this notation, $P_{i}$ are the Planck integrals, given by

$$
P_{i}(T)=\int_{\nu_{i}}^{\nu_{i+1}} B(\nu, T) \mathrm{d} \nu
$$

the parameters $\beta, \gamma$ and $\delta_{i}$ are given by

$$
\begin{aligned}
& \beta= \begin{cases}\frac{1}{3} & \mathrm{SP}_{1} \\
\frac{3}{5} & \mathrm{SP}_{2}, \mathrm{GSP}_{2}\end{cases} \\
& \gamma= \begin{cases}\frac{2}{3} \cdot \frac{1+3 r_{2}}{1-2 r_{1}} & \mathrm{SP}_{1} \\
\frac{4}{5} \cdot \frac{1+3 r_{2}}{1-4 r_{3}} & \mathrm{SP}_{2}, \mathrm{GSP}_{2}\end{cases} \\
& \delta_{i}= \begin{cases}\sum_{j \in J(i)} \frac{P_{j, b}}{\kappa_{j}} & \mathrm{SP}_{1} \\
\sum_{j \in J(i)}\left[\frac{P_{j}}{\kappa_{j}}+\frac{6}{5} \cdot \frac{1-2 r_{1}}{1-4 r_{3}} \frac{P_{j, b}-P_{j}}{\kappa_{j}}\right] & \mathrm{SP}_{2}, \mathrm{GSP}_{2}\end{cases}
\end{aligned}
$$

and the index sets $I$ and $J$ are given by

$$
\begin{gathered}
I= \begin{cases}\{1, \ldots, n\} & \mathrm{SP}_{1}, \mathrm{SP}_{2} \\
\{1\} & \mathrm{GSP}_{2}\end{cases} \\
J(i)= \begin{cases}\{i\} & \mathrm{SP}_{1}, \mathrm{SP}_{2} \\
\{1, \ldots, n\} & \mathrm{GSP}_{2}\end{cases}
\end{gathered}
$$

It should be noted that the quotients

$$
\frac{\sum_{j \in J(i)} \frac{P_{j}^{\prime}}{\kappa_{j}^{3}}}{\sum_{j \in J(i)} \frac{P_{j}^{\prime}}{\kappa_{j}}} \text { and } \frac{\sum_{j \in J(i)} \frac{P_{j}^{\prime}}{\kappa_{j}^{2}}}{\sum_{j \in J(i)} \frac{P_{j}^{\prime}}{\kappa_{j}}}
$$

reduce to $\kappa^{-2}$ and $\kappa^{-1}$, respectively, for $\mathrm{SP}_{1}$ and $\mathrm{SP}_{2}$, as $J(i)=\{i\}$. Because of the significant effort the computation of all Planck integral derivatives $P_{j}^{\prime}$ causes, this substitution is essential for an efficient implementation of the non-averaged models, and at the same time the most significant bottleneck of the $\mathrm{GSP}_{2}$ model. In our implementation, we used a semiimplicit discretization that computed the flux equations based on the temperatures from the previous step.

While the WSGG model reduction (and POD model reduction we have presented so far) was done on the $\mathrm{SP}_{1}$ equations for simplicity, the frequency-averaged model reduction was implemented for the $\mathrm{SP}_{2}$ equations, as $\mathrm{GSP}_{1}$ is identical to the Rosseland approximation and therefore of much lower accuracy than $\mathrm{SP}_{1}[12,17]$. In order to compare approximation quality and numerical effort of $\mathrm{GSP}_{2}$ to $\mathrm{POD}$, a $\mathrm{SP}_{2}$-based variant of $\mathrm{POD}$ was also implemented (the differences of $\mathrm{SP}_{1}$ and $\mathrm{SP}_{2}$ are only marginal, as can be seen from the equations in the previous subsection, so that there should arise no need for a detailed discussion of $\mathrm{SP}_{2}$-based POD).

The first plot in figure 8 shows the approximation error of our $\mathrm{SP}_{2}$-based POD implementation. In general, the approximation is not quite as good as for $\mathrm{SP}_{1}$, as the $\mathrm{SP}_{2}$ model yields solutions with higher variance in space, which is harder for POD to approximate (as was already seen for boundary layers above). The second plot in figure 8 shows the corresponding error for $\mathrm{GSP}_{2}$. The approximation is significantly worse. 

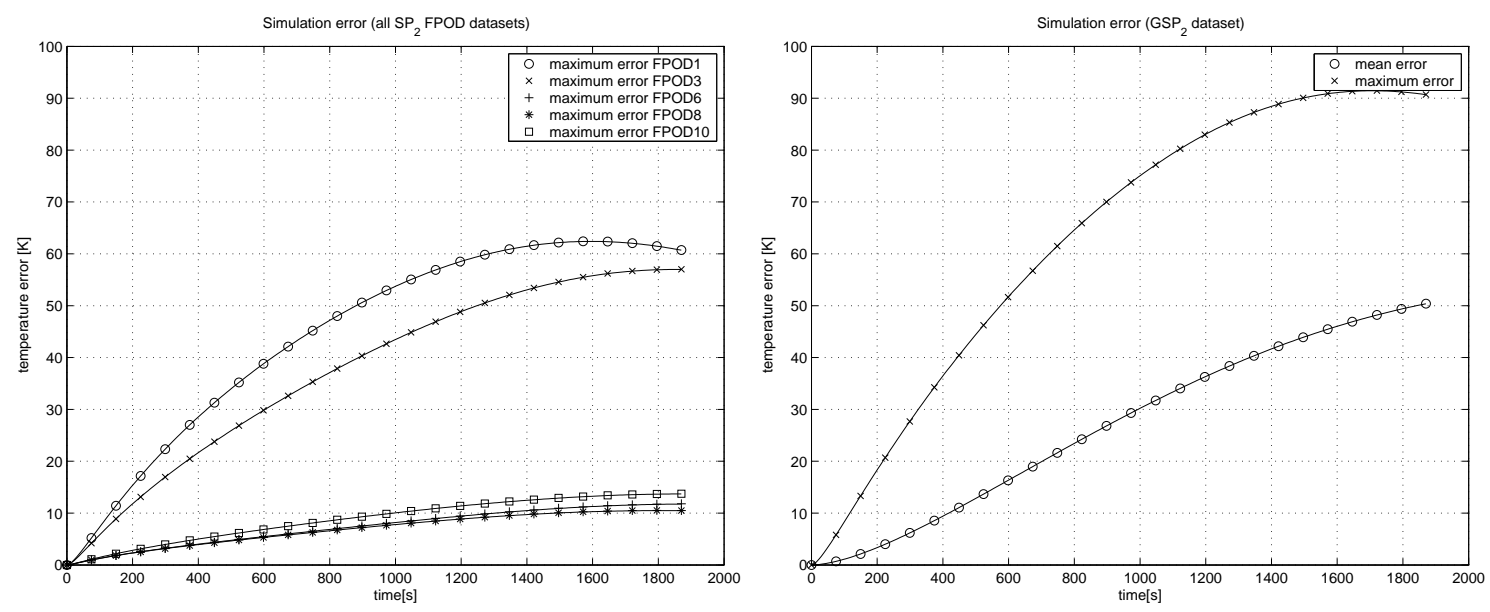

Figure 8: Approximation error of $\mathrm{SP}_{2}$ and $\mathrm{GSP}_{2}$ model

\section{Comparison of the three frequency averaging techniques}

When comparing the numerical effort of WSGG and POD, both methods show advantages and disadvantages. POD requires a solution of the full 283-band system; on the other hand, WSGG requires only a parameter fit, which is, although highly nonlinear, computationally cheaper. The WSGG calculations were done using a WSGG model consisting of ten grey gases, so that there were no advantages in computation time on the side of WSGG, because ten was also the highest numbers of bands used for POD. On the other hand, POD achieves much better results, as long as the number of artificial frequency bands is high enough. For POD models consisting of less than six bands, relatively large temperature errors were encountered at the boundary of the medium; this seems to indicate that the first frequency bands describe the spectrum in the core of the medium, whereas frequency bands corresponding to radiation modes with lower eigenvalues take care of the boundary effects. The WSGG model end temperature errors differ fundamentally from the POD errors. While POD has large errors at the boundary and gives good results for the core of the medium even for low number of bands, WSGG shows low errors at the boundary and large errors in the core.

Further, it is interesting to investigate whether $\mathrm{GSP}_{2}$ or POD lead to better approximation; in order to be as fair as possible, a single band POD should be used in this comparison. Even single band POD performs significantly better than $\mathrm{GSP}_{2}$, and even POD with six bands still outperforms $\mathrm{GSP}_{2}$ with respect to both, accuracy and CPU time requirements.

\section{Conclusion}

In this paper, we presented a new model reduction method for simulating temperature and radiation in hight temperature processes. We showed that, using this new method, significantly better results can be obtained with similar or less numerical effort (if the full solution of the system required for POD is not taken into account, as this is necessary only once and the POD models generated can be used for many simulations). POD does not require special engineering knowledge, as is the case for WSGG; POD can be used as a fully automatic black box algorithm for model reduction, requiring no 
user interaction at all. Even more interesting, POD was also able to outperform $\mathrm{GSP}_{2}$, which has a much stronger theoretical background.

\section{Acknowledgments}

This work has been supported by the Kaiserslautern Excellence Cluster Dependable Adaptive Systems and Mathematical Modeling, by the DFG via SFB 568 and project PI 408/3-1, as well as by the DAAD program PPP Kanada.

\section{References}

[1] K. Afanasiev, M. Hinze, Adaptive control of a wake flow using proper orthogonal decomposition, in Shape optimization and optimal design (Cambridge, 1999), vol. 216 of Lecture Notes in Pure and Appl. Math., Dekker, New York, 2001, pp. 317-332.

[2] V. I. Agoshrov, C. Bardos, Optimal Control Approach in Inverse Radiative Heat Transfer Problems: The Problem of the Boundary Function, 5, ESAIM: Control Optimisation and Calculus of Variations, pp. 259-278, (2000)

[3] V. R. Algazi, D. J. SAkrison, On the optimality of the Karhunen-Loève expansion, IEEE Trans. Information Theory, IT-15 (1969), pp. 319-320.

[4] M. K. Choudhary, N. T. HufF, Mathematical modeling in the glass industry: An overview of status and needs, Glastech. Ber. Glass Sci. Technol., 70:363-370, 1997.

[5] M. Herty, R. Pinnau, M. Seaid, On Optimal Control Problems in Radiative Transfer, preprint, 2005

[6] M. Hinze, S. VolKWeIn, Error estimates for abstract linear-quadratic optimal control problems using proper orthogonal decomposition, Technical Report IMA02-05, KFU Graz, (2005).

[7] H. Hoetelling, Simplified calculation of principal component analysis, Psychometrica, 1 (1935), pp. 27-35.

[8] K. Karhunen, Zur Spektraltheorie stochastischer Prozesse, Ann. Acad. Sci. Fennicae. Ser. A. I. Math.-Phys., 1946 (1946), p. 7.

[9] A. Klar, J. Lang, M. SeaïD, Adaptive solutions of $\mathrm{SP}_{n}$-Approximations to Radiative Heat Transfer in Glass [available as PDF from the authors]

[10] K. KUnisCh, S. VOLKWEIn, Galerkin proper orthogonal decomposition methods for parabolic problems, Numer. Math., 90 (2001), pp. 117-148.

[11] E. W. Larsen, G. Thömmes, A. Klar, M. SeaïD,T. GötZ, Simplified $P_{N}$ approximations to the equations of radiative heat transfer and applications, Journal of Computational Physics, 183:652-675, 2002.

[12] E. W. Larsen, G. Thömmes, A. Klar, Frequency Averaged Approximations to the Equations of Radiative Heat Transfer in Glass, SIAM J. Appl. Math, 64(2):565-582, 2003. 
[13] M. Loeve, Fonctions aleatoire de second ordre, Revue, 48 (1946), pp. 195-206.

[14] S. MANSERVISI, K. HeUSERMAnN, On some optimal control problems for the heat radiative transfer equation, 5, ESAIM: Control, Optimisation and Calculus of Variations, pp 425-444, (2000)

[15] H. Matthies, M. Meyer, Nonlinear galerkin methods for the model reduction of nonlinear dynamical systems, Computers \& Structures, 81 (2003).

[16] M. Meyer, H. Matthies, Efficient model reduction in nonlinear dynamics using the Karhunen-Loève expansion and dual-weighted-residual methods, Computational Mechanics, 31 (2003).

[17] M. F. Modest, Radiative Heat Transfer. Academic Press.

[18] K. Pearson, On lines and planes of closest to points in space, Philosophical Magazine, 2 (1901), pp. 609-629.

[19] R. Pinnau, G. Thömmes, Optimal boundary control of glass cooling processes, M2AS, 120:1261-1281, 2004.

[20] R. PInnau, Analysis of Optimal Boundary Control For Radiative Heat Transfer Modelled by the $\mathrm{SP}_{1}$ System, preprint 2005.

[21] R. Pinnau, A. Schulze, Newton's Method for Optimal Temperature-Tracking of Glass Cooling Processes, to appear in IPSE, 2006.

[22] C. W. RowLEY, Model reduction for fluids, using balanced proper orthogonal decomposition, Internat. J. Bifur. Chaos Appl. Sci. Engrg., 15 (2005), pp. 997-1013.

[23] M. SeaïD, A. Klar, R. Pinnau, Numerical Solvers for Radiation and Conduction in High Temperature Gas Flows, Flow, Turbulence and Combustion, 3, pp 413-432, 2005

[24] M. Seaid, M. Frank, A. Klar, R. Pinnau, G. Thömmes, Efficient numerical methods for radiation in gas turbines, J. Comput. Appl. Math., 170(1):217-239, 2004.

[25] L. Sirovich, Turbulence and the dynamics of coherent structures. I-III, Quart. Appl. Math., 45 (1987), pp. 561-590.

[26] G. Thömmes, R. Pinnau, M. Seaid, T. Götz, A. Klar, Numerical methods and optimal control for glass cooling processes, TTSP, 31(4-6):513-529, 2002. 